\title{
O diálogo entre a Corte Internacional de Justiça e outros Orgãos Judiciais Internacionais sobre questões processuais
}

Loris Marotti*

\section{INTRODUÇÃO}

Este trabalho analisa e discute o diálogo judicial relacionado a questões processuais e, em particular, evidencia o essencial papel desempenhado pela Corte Internacional de Justiça (CIJ) na determinação e no desenvolvimento de princípios e regras de processo judicial internacional. Uma análise quantitativa e qualitativa das referências mais relevantes feitas por órgãos judiciais internacionais às decisões da Corte em questões de procedimento parece confirmar este papel essencial da CIJ, ao menos relativo a princípios e regras fundamentais que são comuns a qualquer procedimento judicial e que são enraizados na própria natureza da função judicial (e.g. os princípios jura novit curia, competence-competence, a disposição do ônus da prova e assim por diante).

Esta análise irá igualmente ilustrar como a CIJ cada vez mais envolve-se em diálogos com outros órgãos judiciais ou quase-judiciais. Quando este diálogo implica a extensão da aplicação de normas e princípios elaborados em diferentes contextos, revela-se um significante processo de mútua fertilização cruzada entre diferentes cortes e tribunais ${ }^{1}$. A este respeito, particular atenção será prestada ao princípio da igualdade processual das partes, o qual foi objeto de uma interessante abordagem reconstrutiva baseada em direitos humanos pela Corte em uma recente opinião consultiva (a "Opinião Consultiva IFAD") ${ }^{2}$. De maneira interessante, os entendimentos da Corte neste casos podem ser observados como expressão de uma tendência geral recente de abertura a valores protegidos por diferentes áreas do direito internacional.

* Università per Stranieri di Perugia 
A primeira parte deste trabalho irá afrontar alguns aspectos gerais relativos à prática de "diálogo judicial" no contencioso internacional e as fontes do direito processual internacional. A segunda parte é uma análise da práxis de diversos órgãos judiciais internacionais que "dialogaram" com a CIJ em questões processuais. A terceira e última parte focará no posicionamento tomado pela CIJ em relação à determinação e interpretação de princípios processuais e, à luz da abordagem adotada pela Corte na Opinião Consultiva IFAD, traçar os possíveis desenvolvimentos emergentes deste tipo de abordagem.

\section{DiÁlOGO JUDICIAL E QUESTÕES PROCESSUAIS NO CONTENCIOSO INTERNACIONAL}

Nos termos do parágrafo 1 (d) do Artigo 38 do Estatuto da Corte Internacional de Justiça, "sob ressalva da disposição do art. 59”, decisões judiciais são consideradas apenas como "meios subsidiários para a determinação das regras de direito". Portanto, a doutrina do stare decisis - através da qual cortes são compelidas pelos seus julgamentos anteriores, e precedentes são reconhecidos como uma fonte (e não mera evidência) do direito - é claramente excluída pelo Artigo 593. De maneira geral, é possível afirmar que esta doutrina não integra o direito internacional vez que nenhum outro tribunal internacional a aplica ${ }^{4}$. Entretanto, como Mohamed Shahabudden apontou em seu magistral trabalho sobre o precedente na Corte Internacional, "o fato que a doutrina do precedente obrigatório não se aplica significa que as decisões da Corte não são precedentes vinculantes; isto não significa que elas não sejam 'precedentes"'5. Em verdade, a CIJ, como qualquer outra corte permanente, tende em levar em consideração sua jurisprudência através de constantes referências à decisões anteriores portanto desenvolvendo uma jurisprudence constante ou o que foi definido como uma "de facto case law"6. Como observado por um ex-Presidente da CIJ, Gilbert Guillaume, o recurso a precedente por juízes e árbitros internacionais possui implicações positivas e negativas ${ }^{7}$. Por um lado, seu uso permite assegurar certeza e igualdade de tratamento aos litigantes e contribui para a organização de um sistema judicial descentralizado através do fortalecimento do diálogo judicial. Por outro lado, o "culto ao precedente" poderia aumentar o risco de imobilização do direito e ser degenerado em um instrumento de governo por juízes.

Para os propósitos deste estudo, salienta-se que o conceito de precedente é proximamente similar aquele de "diálogo judicial". De fato, este é frequentemente utilizado para descrever a prática seguida por cortes e tribunais internacionais de usar e interagir com a jurisprudência de outros tribunais. Em outras palavras, este conceito expressa o recurso ao "precedente externo" por juízes internacionais ${ }^{8}$.

Somente em tempos recentes a doutrina internacional iniciou a refletir sobre esta específica questão. Isto não surpreende se se considera que o fenômeno parece ser estritamente relacionado à relativamente recente proliferação de 
organismos judiciais internacionais9. Diálogo internacional no contencioso internacional, em sua conotação positiva, tem sido observado como um valoroso instrumento para enfrentar problemas que emergiram da multiplicação de instâncias jurisdicionais e, ao menos "acalmar os medos daqueles preocupados com a fragmentação do direito internacional"10. A prática de engajar-se em tal interação jurisprudencial, portanto, tem representado a principal solução para escapar da condição de isolamento formal na qual organismos judiciais naturalmente operam ${ }^{11}$.

Um ponto que merece ser considerado refere-se às técnicas adotadas por estes órgãos judiciais para justificar e legitimar o recurso à decisões externas. Nesse sentido, deveria parecer claro que - dada a ausência de qualquer doutrina de obrigatoriedade do precedente e a ausência de uma hierarquia ordenando o "sistema judicial" internacional - estas técnicas de diálogo não são previstas por qualquer dispositivo estatutário ou instrumento positivo. Elas são simplesmente o resultado das atividades argumentativas e interpretativas executadas pelas cortes. Por exemplo, entre as possíveis justificativas para o uso de decisões de outros tribunais internacionais, tem-se feito referência à regra contida no parágrafo 1(c) do Artigo 31 da Convenção de Viena sobre Direito dos Tratados (CVDT), especialmente em relação à interpretação dos instrumentos contitutivos e regulamentos de cortes internacionais. Como se sabe, esta regra estabelece que, para a interpretação de um tratado “(...) serão levados em consideração, juntamente com o contexto (...) quaisquer regras pertinentes de Direito Internacional aplicáveis às relações entre as partes". Um exemplo deste modus operandi pode ser encontrado no julgamento da Corte Europeia de Direitos Humanos (CEDH) no caso Mamatkulov and Abdurasulovic v. Turkey ${ }^{12}$. Neste caso, a Corte reconheceu a natureza obrigatória de suas medidas cautelares e, ao fazê-lo, fez referência à práxis do Comitê de Direitos Humanos, da Corte Interamericana de Direitos Humanos, a Comissão de Direitos Humanos e à Corte Internacional de Justiça ${ }^{13}$.

Antes de analisar a jurisprudência destes tribunais, a CEDH mencionou o Artigo 31, (3), (c) para estabelecer que a Convenção Europeia de Direitos Humanos "(...) deve ser interpretada tanto quanto possível de maneira consistente com outros princípios do direito internacional do qual ela forma parte"14. Subsequentemente, a mesma Corte adicionou que "é de crucial importância que a Convenção seja interpretada e aplicada de maneira a assegurar seus direitos práticos e efetivos, não teóricos ou ilusórios. Uma falha da Corte em manter uma abordagem dinâmica e evolutiva arriscaria, de fato, transformá-a num documento que precisaria de reforma e melhoramento" 15 . Contudo, pode-se questionar se o recurso a um instrumento formal, como o Artigo 31 da CVDT, pode oferecer uma adequada base legal de maneira a justificar o uso de precedentes judiciais externos. Isso é especialmente verdade se se considera que a aplicação da regra interpretativa contida no Artigo 31, (3), (c), sujeita à fonte externa, como um tratado, é aplicável to todas as partes de uma controvérsia ${ }^{16}$. 
No campo do processo international, excluindo-se estas técnicas derivadas da interpretação de tratados, os métodos mais utilizados por cortes internacionais para fazer recurso à prática judicial externa é a utilização de princípios gerais do direito. Com efeito, de maneira a encontrar, enunciar e aplicar um determinado princípio processual, uma corte internacional tende a demonstrar a larga aceitação deste no direito internacional referindo-se à prática internacional e, em particular, como se verá, à jurisprudência da CIJ relacionada à normas procedimentais básicas. Por exemplo, no caso US - Shirts and Blouses, o Órgão de Apelação da OMC, determinando a posição do ônus da prova, decidiu que “(...) diversos tribunais internacionais, incluindo a Corte Internacional de Justiça, aceitaram e aplicaram de maneira geral e consistente a regra que a parte que alega um fato, seja o requerente ou o requerido, é responsável por prová-lo. Igualmente, é um cânone probatório aceito de maneira geral na civil law, common law e, de fato, maior parte das jurisdições, que o ônus da prova compete à parte, seja reclamante ou reclamado, que alega uma determinada tese de acusação ou defesa" ${ }^{17}$.

Para melhor explicar este aspecto, faz-se necessário acrescentar algumas palavras no que parece ser a mais nevrálgico questão deste tópico, nomeadamente a questão de fontes do direito internacional processual. Neste sentido, parece evidente que o ponto de partida de qualquer discussão sobre fontes do direito internacional está encrustado no Artigo 38 (1) do Estatuto da CIJ, que serve igualmente como uma lista de fontes do direito processual internacional ${ }^{18}$. De maneira interessante, além dos instrumentos constitutivos e das regras de procedimento de tribunais internacionais - as quais representam as fontes (escritas) primárias -, parte autoritativa da doutrina, também à luz das indicações delineadas na prática judicial international, parece condividir a visão que existe um conjunto de normas processuais fundamentais possuindo um caráter básico e residual com uma aplicação geral. Afirma-se que estas regras devem ser respeitadas, mesmo quando elas não são necessariamente incorporadas em disposições estatutárias ${ }^{19}$. Não obstante, autores não concordam em relação às fontes das quais estas normas originariam $^{20} \mathrm{e}, \mathrm{em}$ geral, quanto à sua natureza ${ }^{21}$.

O que deve ser sublinhado, contudo, é que aparentemente decisões judiciais - especialmente decisões da CIJ - vem ganhado um papel proeminente no interior do sistema de fontes de normas processuais fundamentais, vez que elas representam um canal privilegiado para a "abstração" destas fontes. Em outras palavras, embora seja geralmente claro que no campo do direito processual internacional, excetuando as normas estatutárias, exista uma correspondência entre as fontes listada no Artigo 38, (1), do Estatuto da CIJ (i.e., ocorre que as mesmas normas procedimentais podem ser igualmente referenciadas à prática costumeira, princípios gerais do direito ou decisões judiciais) a crescente prática de referir-se à jurisprudência da CIJ em matérias de princípios fundamentais processuais é indicador do papel primário que a Corte assumiu na verificação destes princípios. Portanto, estas normas parecem encontrar suas fontes nos 
"princípios gerais de direito" (sejam eles princípios do direito internacional ou princípios in foro domestico), dado à ativa, e frequentemente ampla, função discricionária outorgada ao intérprete para a sua dedução ${ }^{22}$. Portanto, podese afirmar que o diálogo judicial, seja implícito ou explícito, especialmente na área do direito processual, passa primordialmente através do canal dos princípios gerais do direito, cuja identificação através do recurso à práxis externa é bastante usual.

\section{O DIÁlOGO ENTRE CORTES E O PAPEL PREDOMINANTE DA CORTE INTERNACIONAL DE JUSTIÇA EM QUESTÕES PROCESSUAIS}

Como mencionado previamente, a CIJ não adota uma doutrina formal do respeito ao precedente, "exceto talvez em matéria de procedimento"23. É igualmente reconhecido que a Corte sempre foi relutante em citar ou referenciarse à jurisprudência de outros tribunais, embora tenha demonstrado recentemente grande abertura nesse sentido ${ }^{24}$.

Entretanto, é digno de nota que a primeira manifestação de um diálogo judicial em que a Corte participou referia-se a uma questão procedimental. No caso Polish Postal Service in Danzig, a Corte Permanente de Justiça Internacional mencionou a sentença arbitral Pious Funds of the Californias quando enfrentou o princípio da res judicata ${ }^{25}$.

De maneira similar, no caso Nottebohm (objeção preliminar) a CIJ, pela primeira vez, citou uma decisão externa, nomeadamente a bem conhecida sentença arbitral do caso Alabama. Sublinhando que o princípio da compétence de la compétence é aceito pelo direito internacional geral, a Corte apontou que

\footnotetext{
"Desde o caso Alabama, tem sido reconhecido de maneira geral [...] que, na ausência de um acordo em contrário, um tribunal internacional possui o direito de decidir sua própria jurisdição e possui o poder de interpretar, para este propósito, os instrumentos que governam esta jurisdição"26.
}

Além destes dois casos, a Corte jamais referiu-se à jurisprudência externa em relação a questões procedimentais. Em verdade, preocupou-se sempre em manter consistência judicial neste campo referindo-se o máximo possível à própria jurisprudência.

Ao contrário, outros tribunais internacionais referem-se frequentemente à jurisprudência da $\mathrm{CIJ}$ (ou da CPJI) especialmente quando estão enfrentando questões procedimentais básicas. Pode-se referir a diversos exemplos para ilustrar esta tendência. 
No acima referenciado caso US - Shirts and Blouses, o Órgão de Apelação da $\mathrm{OMC}$ fez referimento à prática da $\mathrm{CIJ}$ em determinar o conteúdo da regra da disposição do ônus da prova, de acordo com o qual a parte que alega um fato é responsável por provar-lhe $e^{27}$.

Esta passagem foi amplamente citada em relatórios subsequentes do Órgão de Apelação. Por exemplo, no caso European Communities - Tariff Preferences, o mesmo órgão mencionou à referenciada decisão ${ }^{28}$, acrescentando que "de maneira consistente ao princípio jura novit curiae, não é responsabilidade das Comunidades Européias fornecer-nos a interpretação legal a ser dada a um determinado dispositivo na presente cláusula" ${ }^{29}$. Neste ponto, o Órgão de Apelação explicitamente citou o caso Nicaragua ao articular o conteúdo do princípio jura novit curiae ${ }^{30}$.

Diverso exemplo oriundo da jurisprudência da OMC pode ser encontrado no caso United States - Anti-Dumping Act of 1916, no qual o Órgão de Apelação, lidando com o princípio compétence de la compétence notou que "[...] é uma regra largamente aceita que um tribunal internacional é competente a considerar a questão de sua própria jurisdição por sua própria iniciativa, e satisfazer a si mesmo se possui jurisdição em qualquer caso que surja perante a si mesmo". Portanto, fez extensiva referência à prática da $\mathrm{CPJI}$ e da $\mathrm{CI}{ }^{31}$.

No que tange à prática do Tribunal Penal Internacional para antiga Iugoslávia (TPIAI), a decisão da Corte de Apelação no caso Allegations of Contempt Against Prior Counsel, Milan Vujin (Prosecutor v. Tadic) 32 é particularmente relevante. Com efeito, ao determinar se possuía os poderes para lidar com o caso, o tribunal extraiu da jurisprudência da CIJ (precisamente citada neste caso também $)^{33}$ uma passagem de acordo com a qual o tribunal "possui uma jurisdição inerente, derivante de sua função judicial, de assegurar que sua jurisdição, expressamente prevista por aquele Estatuto, não seja frustrada e que sua básica função judicial seja salvaguardada" ${ }^{34}$.

A Corte Interamericana de Direitos Humanos (CIDH) demonstra igualmente uma frequente tendência em dialogar com a CIJ em questões procedimentais básicas. Por exemplo, no caso Fairén-Garbi and Solís Corrales v. Honduras (mérito), a Corte referiu-se aos casos Corfu Channel e Nicaragua para demonstrar que "a jurisprudência internacional reconheceu o poder de cortes de avaliar provas livremente, embora ela tenha sempre evitado uma regra rígida relativa ao montante de prova necessária para dar suporte à decisão"35. Ainda, no caso Velásquez-Rodriguez v. Honduras, a Corte referenciou-se ao caso Lotus da CPJI ao mencionar o princípio jura novit curia "[...] no qual a jurisprudência internacional repetidamente embasou-se e que estabelece que a Corte possui o poder e o dever de aplicar as regras jurídicas relevantes em um caso, mesmo quando as partes não as invocaram expressamente"36.

Voltando-se ao Tribunal Internacional sobre Direito do Mar (ITLOS), é possível identificar diversas ocasiões em que este diálogo estabeleceu-se em sua 
jurisprudência. No caso "Grande Prince", o Tribunal expressamente citou uma passagem de uma decisão da CIJ no caso Appeal Relating to the Jurisdiction of the ICAO Council para confirmar que ele "possui o direito de lidar com todos os aspectos da questão da jurisdição, tendo eles sido expressamente solevados pelas partes ou não"37.

Ainda mais interessante para os objetivos deste trabalho é o julgamento emanado pelo ITLOS em 28 de Maio de 2013 nos casos $M / V$ "Louisa"38. Nesta decisão, o Tribunal enfrentou diversas questões de natureza procedimental e fez referência à prática da $\mathrm{CIJ}$ a respeito.

Por primeiro, tendo as partes divergido em relação à finalidade da jurisdição do Tribunal por suas declarações feitas sob o Artigo 287 da Convenção das Nações Unidas sobre o Direito do Mar (UNCLOS), o Tribunal estabeleceu, citando uma passagem do caso Certain Norwegian Loans que, "[...] em casos onde os Estados Parte fizeram declarações com diferentes finalidades sob o Artigo 287 da Convenção, sua jurisdição existe somente na extensão em que a substância das declarações das duas partes coincide" ${ }^{39}$.

Segundo, o Tribunal considerou ser apropriado referir-se à "estabelecida jurisprudência da CIJ de acordo com a qual 'compete ao Requerente, em sua Inicial, apresentar à Corte a disputa que deseja a decisão da Corte e estabelecer os pedidos que está submetendo a ela"' 40 .

Terceiro, ITLOS referiu-se ao julgamento no caso Oil Platform para demonstrar que "para permitir que o tribunal determine se ele possui jurisdição, ele deve estabelecer uma ligação entre os fatos alegados por São Vicente e Granadinas e os dispositivos da Convenção por este Estado alegados e demonstrar que tais dispositivos podem sustentar o pedido ou pedidos submetidos por São Vicente e Granadinas" ${ }^{\prime 4}$.

Quarto - considerando que neste caso Espanha argumentou que São Vicente e Granadinas tentou mudar a natureza da controvérsia apresentado um novo pedido baseado no Artigo 300 da Convenção apenas depois do encerramento da fase escrita do procedimento - o Tribunal observou que "é um requerimento legal de qualquer novo pedido para sua admissão que ele seja levantado na petição inicial ou esteja nela implícito. (...) Conclui-se do supra referido, enquanto que os pedidos subsequentes podem aclarar os termos da inicial, eles não podem ir além dos limites do pedido como estabelecidos na petição inicial" ${ }^{42}$. Portanto, o Tribunal referiu-se largamente à jurisprudência da CPJI e da CIJ na interpretação dos dispositivos de seus Estatutos e Regulamentos que correspondiam àqueles do ITLOS, através do qual o Tribunal inferiu a conclusão supra mencionada.

Embora não seja possível aqui cobrir a práxis de todos os órgãos judiciais que dialogaram com a Corte em matérias procedimentais, parece claro do atual exame que o que vem sublinhado por autoritativos autores é efetivamente confirmado pela práxis ${ }^{43}$. Em substância, esta prática demonstra que a Corte 
Internacional desempenhou e desempenha um papel central neste campo. Seu corpo de jurisprudência processual representa um sólido ponto de referência para outras cortes e, até o momento, continua a contribuir ao aumento do grau de homogeneidade entre órgãos judiciais, ao menos no que se refere àquelas básicas ou essenciais normas processuais ${ }^{44}$.

\section{DESENVOLVIMENTOSRECENTESRELATIVOSÀABORDAGEM RECONSTRUTIVA DA CIJ: A "OPINIÃO CONSULTIVA IFAD"}

Foi já sublinhado que a Corte tradicionalmente demonstra relutância em referi-se à jurisprudência externa, ou à fontes externas ${ }^{45}$, especialmente em relação à determinação e interpretação de princípios processuais internacionais. Contudo, como mencionado previamente, a prática recente é um indicador de uma atitude distinta assumida pela CIJ, a qual tem se abrido de maneira crescente à decisões e fontes externas. Assim, da perspectiva da Corte, diálogo judicial tem gradualmente perdido seu caráter de "diálogo unidrecional", sendo a própria Corte progressivamente envolvida em interação externa.

Tal tendência pode ser igualmente reconhecida no que se refere à princípios gerais de direito processual, como claramente demonstrado pela recente opinião consultiva no caso Judgment No. 2867 of the Administrative Tribunal of the International Labour Organization upon a Complaint Filed against the International Fund for Agricultural Development, (doravante "Opinião Consultiva IFAD” ${ }^{46}$. Neste caso a Corte, verificando a finalidade da aplicação do princípio da igualdade das partes, adotou uma abordagem muito interessante que merece ser analisada à luz das observações supra mencionadas.

Neste caso, a Corte pela quinta vez atuou como um órgão de revisão para julgamentos de tribunais administrativos de organizações internacionais ${ }^{47}$. Esta peculiar função - que até 1995 era também estabelecida para as decisões do Tribunal Administrativo das Nações Unidas (TANU) ${ }^{48}$ - é atualmente fornecida apenas pelo Estatuto pelo Tribunal Administrativo da Organização Internacional do Trabalho (TAOIT) ${ }^{49}$ e apresenta uma característica controversa que afeta a igualdade das partes seja no acesso à CIJ quanto ao procedimento perante ela. Em verdade, por um lado, o Estatuto do TAOIT não permite que o indivíduoempregado tenha acesso à possibilidade de revisão dos julgamentos do TAOIT pela CIJ, sendo a organização-empregadora a única parte permitida a fazê-lo; por outro lado, no que se refere à ausência de igualdade no procedimento perante a Corte, esta última, já em 1956, sublinhou que tal desigualdade "decorre (...) dos dispositivos do Estatuto da Corte" ${ }^{50}$. Efetivamente, sob o artigo 66 do Estatuto da Corte, apenas Estados e organizações internacionais, e não indivíduos, podem submeter considerações escritas e orais à Corte. Em outras palavras, membros do staff não possuem o direito de participar no procedimento de revisão, embora o último seja conduzido apenas formalmente na forma consultiva ${ }^{51}$. 
$\mathrm{Na}$ Opinião Consultiva IFAD, a Corte explorou a oportunidade para lidar com diversas questões do princípio da igualdade das partes. E o fez à luz da "evolução" que o princípio da igualdade das partes experimentou ao longo dos anos.

Em particular, a CIJ tomou como ponto de referência para a determinação do conteúdo do princípio no caso em comento os dois Comentários Gerais adotados pelo Comitê de Direitos Humanos ao Artigo 14, para. 1, do Pacto Internacional sobre Direitos Civis e Políticos de 1966 (PIDCP). O Artigo 14, para. 1 , estabelece que "todas as pessoas são iguais perante os tribunais e as cortes de justiça”. A Corte notou que o conteúdo mais detalhado do segundo comentário adotado em 2007 indica uma renovada conotação do princípio. Ademais, a Corte observou que “(...) se direitos processuais são conferidos, eles devem ser conferidos à todas as partes a menos que distinções possam ser justificadas através de argumentos objetivos e razoáveis" 52 . Portanto, o princípio da igualdade das partes, que deriva das exigências de boa administração da justiça ${ }^{53}$, “(...) deve ser ora entendido como incluindo o acesso, numa base igualitária, à apelação ou a à remédios judiciais similares, a menos que uma exceção possa ser invocada com argumentos objetivos e razoáveis" ${ }^{\prime 5}$.

Neste sentido, enquanto a Corte tenha admitido que não poderia ter agido para retificar a "estrutural" ausência de igualdade no acesso à Corte (não estando "em posição de reformar este sistema" ${ }^{55}$ ), ela tentou aliviar a desigualdade no procedimento utilizando-se de dois expedientes processuais. Por primeiro, ela decidiu que o presidente do Fundo deveria transmitir à Corte qualquer alegação escrita que a parte da controvérsia original, o membro do staff, tenha desejado trazer à atenção da Corte. Segundo, a Corte decidiu que não haveria fase oral $^{56}$. De acordo com a CIJ, tais medidas teriam sido suficientes para "aliviar substancialmente" a desigualdade no procedimento e considerar "não suficientemente persuasivas" as razões que poderiam levá-la à evitar a emanação da opinião consultiva ${ }^{57}$.

A abordagem adotada pela Corte na identificação do conteúdo do princípio da igualdade das partes contém novos elementos relevantes. Com efeito, ao interpretar o conteúdo deste princípio, a Corte levou em consideração a evolução de seu significado ao longo do tempo. Significativamente, a Corte estabeleceu um diálogo com um órgão "não-judicial", o Comitê de Direitos Humanos, considerando-o como um intérprete autoritativo do Artigo 14, para. 1, do PIDCP. Como mencionado, a CIJ explicitamente referiu-se aos Comentários Gerais adotados por este órgão para deduzir e atualizar o conteúdo do princípio fundamental em questã ${ }^{58}$. Apesar deste diálogo, a CIJ mostrou que que qualquer envolvimento de um indivíduo no contencioso internacional implica que o princípio da igualdade das partes não poder ser aplicado a partir de uma mera perspectiva interestatal (o que conecta suas raízes ao princípio de soberania). Sua aplicação requer que devida consideração seja prestada ao desenvolvimento de direitos processuais fundamentais inseridos na maioria dos instrumentos de direitos humanos. Em substância, tal abordagem baseada em direitos humanos 
permitiu a Corte de adaptar e integrar os acima mencionados princípios no interior de contexto de direitos humanos.

Deve ser dito que, para os propósitos deste trabalho, o elemento mais inovador da Opinião Consultiva IFAD não é somente o fato que a Corte dialogou com um órgão de direitos humanos ou referenciou-se a um tratado de direitos humanos $^{59}$, mas o fato que ela assim o fez em relação a um princípio processual fundamental. Com efeito, como já mencionado, a Corte raramente embasou-se expressamente em fontes externas ao aplicar as regras e princípios essenciais de processo. Neste campo, a Corte sempre liderou mas nunca seguiu.

\section{APONTAMENTOS CONCLUSIVOS}

A questão do papel do diálogo judicial no contencioso internacional tem ganhado crescente importância seguindo o desenvolvimento do debate sobre proliferação de órgãos judiciais e quase-judiciais. Este fenômeno é também estritamente coligado a outra interessante questão, nomeadamente a assim chamada "integração de valores" no direito internacional ${ }^{60}$. Esta questão aparece como resultado do grau ao qual estes órgãos, cada qual competente numa área específica do direito internacional, são permeáveis a valores protegidos por diferentes áreas, como o direito internacional dos direitos humanos ou direito ambiental ${ }^{61}$. Neste sentido, diálogo judicial como qualquer outro tipo de abertura a regimes externas claramente constitui a mais importante ferramenta para a circulação e a integração destes valores entre cortes.

Parece que, na Opinião Consultiva IFAD, a abordagem da CIJ ao princípio da igualdade das partes possa ser lido como um esforço para integrar o conteúdo deste princípio - o qual pertence a um setor particular do direito internacional, nomeadamente direito processual - a valores emergentes no setor de direitos humanos. Portanto, o diálogo executado pela CIJ no caso em comento - o qual obviamente não pode ser qualificado como "diálogo judicial” num sentido técnico - ocorreu de maneira a atingir tal integração.

Nesta altura, seria possível especular se uma abordagem baseada em direitos humanos em relação a questões processuais, como no caso descrito acima, serviria a trazer posteriores e concretas inovações ao processo perante a CIJ. Efetivamente, parece que esta abordagem poderia haver um impacto em outros aspectos no processo perante a Corte. Poderia-se referir, por exemplo, à questão de participação de indivíduos como amici curiae em casos contenciosos perante a Corte Internacional ${ }^{62}$. Como se sabe, nem o Estatuto nem o Regulamento da Corte contemplam esta particular forma de participação, com exceção do Artigo 34(2) do Estatuto, o qual é limitado à participação de organizações internacionais ${ }^{63}$. Indivíduos podem aparecer perante a Corte apenas como testemunhas ou peritos, e sempre sob o requerimento da Corte ${ }^{64}$. Contudo, o fato que a participação de indivíduos como amici curiae não é expressamente prevista no Estatuto não significa 
que a Corte não poderia aceitar e considerar observações submetidas por indivíduos interessados atuando não meramente na qualidade de testemunha e perito. $O$ Estatuto é simplesmente silente em relação à questão ${ }^{65}$. Efetivamente, como tem sido argumentado, a Corte teria o poder de permitir e aceitar submissões de amici curiae em virtude da autonomia que ela goza em relação a evidência, e o exercício concreto deste poder não estaria submetido a uma emenda formal ao Estatuto ${ }^{66}$. Poderia ser sugerido que a CIJ poderia abrir-se a submissões individuais-amici curiae, ao menos no contexto de controvérsias envolvendo direta ou indiretamente direitos individuais e interesses. Neste sentido, referência a precedentes externos como a práxis das cortes de direitos humanos (a CEDH e a CIDH), poderia ajudar a legitimar a introdução de tais instrumentos processuais no procedimento perante a CIJ. Os Estatutos e Regulamentos destas cortes expressamente preveem a possibilidade de dossiês de amici curiae ${ }^{67}$ para garantir, inter alia, alguma forma de acesso àqueles que não podem atuar como uma parte stricto sensu perante uma corte internacional.

Em conclusão, da análise efetuada pode ser observado que, apesar do fato do diálogo relativo a questões processuais básicas ter sido executado majormente de maneira vertical e unilateral e ter tido a Corte desempenhado um papel fundamental neste campo ao longo dos anos, a mesma Corte demonstrou que mesmo em relação a um bem estabelecido princípio fundamental, como o da igualdade de partes, há ainda espaço para abertura à diferentes valores emergentes de outras áreas do direito internacional e encontrar soluções inovativas. Numa visão ampla, esta análise reafirmou que o diálogo entre órgãos judiciais e/ou quase judiciais é inclinado a favorecer a circulação de valores no direito internacional. A integração de valores emergentes do direito internacional dos direitos humanos no interior do domínio do direito internacional processual representa um maior exemplo deste fenômeno. Como verificado, ao permitir-se ir além da tradicional perspectiva interestatal, esta integração poderia contribuir a revelar novos e ainda não explorados desenvolvimentos no contencioso internacional.

\section{NOTAS}

1 Especialmente em relação aos princípios e regras do processo internacional, é importante rememorar que, já em 2008, BROWN, The Cross-Fertilization of Principles Relating to Procedure and Remedies in the Jurisprudence of International Courts and Tribunals, in Loyola of Los Angeles International and Comparative Law Review, 2008, p. 219 ss., sublinhou a tendência de cortes e tribunais internacionais levar em consideração a prática de outros tribunais em questões processuais e sublinhou a emergência de abordagens comuns neste campo.

2 Corte Internacional de Justiça, Judgment No. 2867 of the Administrative Tribunal of the International Labour Organization upon a Complaint Filed against the International Fund for Agricultural Development, Advisory Opinion of 1 February 2012, in ICJ Reports, 2012, p. 10.

3 Ver SHAHABUDDEN, Precedent in the World Court, Cambridge, 1996.

4 GUILLAUME, The Use of Precedent by International Judges and Arbitrators, in Journal of International Dispute Settlement, 2011, p. 9 ss.; Shaw, International Law, Cambridge, 2008, p. 110.

RDUNO, v. 1, n. 2, Jul - Dez/2018 - pag (49-71) 
5 SHAHABUDDEN, Precedent in the World Court, cit., p. 2, grifo adicionado.

6 REINISCH, The Proliferation of International Dispute Settlement Mechanism: The Threat of Fragmentation vs. the Promise of a More Effective System? Some Reflections From the Perspective of Investment Arbitration, in BUFFARD, CRAWFORD, PELLET, WITTICH (eds.), International Law Between Universalism and Fragmentation. Festschrift in Honour of Gerhard Hafner, Leiden/ Boston, 2008, p. 123. Ver igualmente ROSENNE, The Perplexities of Modern International Law, Leiden, 2004, pp. 44-46.

7 GUILLAUME, The Use of Precedent, cit., p. 23.

8 A expressão "precedente externo" é devida a Guillaume, The Use of Precedent, cit. Diversos autores preferem não utilizar a palavra "precedente" ao abordar a questão. Ver, por exemplo, MILLER, An International Jurisprudence? The Operation of "Precedent" Across International Tribunals, in Leiden Journal of International Law, 2002, p. 488, de acordo com quem “(...) 'Precedent' is a loaded term, especially in international law. (...) To the extent that it is understood to refer to other practices, the term is ambiguous; it means different things in different legal system and can have multiple meanings even within the same system, depending on the context in which it is used [...]". Ver igualmente ZAMMIT BORDA, Precedent in International Criminal Courts and Tribunals, in Cambridge Journal of International and Comparative Law, 2013, p. 288. De maneira geral, é interessante notar que um autor pontuou que o precedente externo possui relevência apenas como um mero "exemplo"; em outras palavras, “(...) it does not indicate the decisional criterion that must be followed in the subsequent cases, but it only shows that a given norm has been applied in a certain way in a certain case. Thus, example informs but does not suggest, nor does it impose a decision consistent with it". Ver TARUFFO, Precedente e giurisprudenza, Napoli, 2007, p. 32 (Tradução em inglês realizada pelo autor).

9 ROMANO, Deciphering the Grammar of the International Jurisprudential Dialogue, in International Law and Politics, 2009, pp. 759-760, observa que "[t]he role of precedent across international courts is still a largely unmapped territory. While most literature to date has focused on the treatment by courts of their own precedents, there have been very few studies about the treatment of precedent across international courts". Entre outras recentes contribuições lidando com este tópico, ver WEINIGER and SCHREUER, Conversations across Cases: Is there a Doctrine of Precedent in Investment Arbitration?, in Transnational Dispute Management, 2008; ZAMMIT BORDA, Precedent in International Criminal Courts, cit.

10 MILLER, An International Jurisprudence? cit., p. 499.

11 Como a Corte Penal para a Antiga Iugoslavia entendeu no caso Prosecutor v. Tadic, Decision on the defence motion for Interlocutory Appeal on Jurisdiction, IT-94-1-AR722, October 1995, para. 11) "[i]nternational law, because it lacks a centralised structure, does not provide for an integrated judicial system operating an orderly division of labour among a number of tribunals, where certain aspects or components of jurisdiction as a power could be centralised or vested in one of them but not the others. In international law, every tribunal is a self-contained system (unless otherwise provided)".

12 ECtHR, Mamatkulov and Abdurasulovic v. Turkey, Applications Nos. 46827/99 and 46951/99, Judgment of 6 February 2003.

13 Ibidem, paras. 102-105, citando LaGrand (Germany v. United States), Judgment of 27 June 2001, in ICJ Reports, 2001, para. 466.

14 Ibidem, para. 99.

165e Court notes that in the light of the general principles of international law, the law of treaties and international case-law, the interpretation of the scope of interim measures cannot be dissociated from the proceedings to which they relate or the decision on the merits they seek to protect".

16 Especificamente em relação ao recurso à fontes externas como tratados não obrigatórios a todas as partes numa controvérsia (portanto, incidindo no sentido do Artigo 31(3)(c) da CVDT), uma 
abordagem interessante comparável à teoria de Taruffo sobre precedentes externos (ver, supra, nota 8), tem sido adotada no interior da jurisprudência da OMC. Por exemplo, no caso European Comunities-MeasuresAffecting the Approval and Marketing of Biotech Products (Reports of the Panel, WT/DS291/R, WT/DS292/R, WT/DS293/R, 29 September 2006), o painel sustentou que "[...] other relevant rules of international law may in some cases aid a treaty interpreter in establishing, or confirming, the ordinary meaning of treaty terms in the specific context in which they are used. Such rules would not be considered because they are legal rules, but rather because they may provide evidence of the ordinary meaning of terms in the same way that dictionaries do. They would be considered for their informative character. $[\ldots][T]$ he mere fact that one or more disputing parties are not parties to a convention does not necessarily mean that a convention cannot shed light on the meaning and scope of a treaty term to be interpreted" (paras. 7.92 and 7.94, emphasis added). Para uma visão geral, ver CANNIZZARO, Il rilievo degli accordi esterni nell'interpretazione degliaccordi OMC, in LIGUSTRO, SACERDOTI (eds.), Problemi e tendenze del diritto internazionale dell'economia. Liber amicorum in onore di Paolo Picone, Napoli, 2011, p. 513 ss.

17 Report of the Appellate Body, US - Measures Affecting Imports of Woven Wool Shirts and Blouses from India, WT/DS33/AB/R, 25 April 1997, p. 14, grifo nosso.

18 O posicionamento de Brown neste ponto de que "[...] if customary international law, general principles of law, judicial decisions and writings of publicists are sources of the substantive rules of international law, there is no reason why they might not also serve as a source of the procedural rules of international law [...]" é bastante convincente. See BROWN, The Cross-Fertilization, cit., p. 37.

19 Ver, entre outros, CHENG, General Principles of Law as Applied by International Courts and Tribunals, London, 1953, p. 257 ss.; SERENI, Principi generali di diritto e processo internazionale, Milano, 1955, passim; SCHLESINGER, Research on the General Principles of Law Recognized by Civilized Nations, in American Journal of International Law, 1957, p. 736; MANI, International Adjudication: Procedural Aspects, The Hague, 1980, p. 12; NEGRI, I principi generali del processo internazionale nella giurisprudenza della Corte internazionale di giustizia, Napoli, 2002, pp. 32-33; KOLB, General Principles of Procedural Law in ZIMMERMANN, TOMUSCHAT, and OELLERS-FRAHM (eds), The Statute of the International Court of Justice: A Commentary, Oxford, 2012, p. 871 ss.

20 MATSCHER, Standing Before International Courts and Tribunals, in EPIL, 1981 p.191; THIRLWAY, Procedure of International Courts and Tribunals, in EPIL, 1997, p. 1128. Ver tambem, do mesmo autor, Procedural Law and the International Court of Justice, in LOWE, FITZMAURICE (eds.), Fifty Years of the International Court of Justice. Essays in Honour of Sir Robert Jennings, Cambridge, 1996, p. 389; and GAFFNEY, Due Process in the World Trade Organization: The Need for Procedural Justice in the Dispute Settlement System, in American University International Law Review, 1999, p. 1179-1180; BROWN, The Cross-Fertilization, cit., pp. 36-37; KOLB, La maxime de la "bonne administration de la justice" dans la jurisprudence internationale, in Lobservateur des Nations Unies, 2009, pp. 7-8.

21 Para uma visão geral ver PALOMBINO, Judicial Economy and the Limitation of the Scope of the Decision in International Adjudication, in Leiden Journal of International Law, 2010, p. 910 ss.

22 A Corte Internacional de Justiça referiu-se a "princípios de direito processual" em ao menos duas ocasiões ao longo de sua história. A primeira foi nos casos South-West Africa (Segunda Fase), Judgment of 18 July 1966, em ICJ Reports, 1966, p. 6, para. 64, no qual a Corte qualificou como um elementar princípio de direito processual “ (...) that a distinction has to be made between, on the one hand, the right to activate a court and the right of the court to examine the merits of the claim, - and, on the other, the plaintiff party's legal right in respect of the subject-matter of that which it claims, which would have to be established to the satisfaction of the Court". A segunda ocasião na qual a Corte fez referência a este conceito foi no caso Land, Island and Maritime Frontier Dispute (Application to Interene), Judgment of 13 September 1990, in ICJ Reports 1990, p. 92, para. 102. Ao enfrentar a questão do Estado requerendo a intervenção, a Corte declarou que este "does not become party to the proceedings, and does not acquire the rights, or become subject to the 
obligations, which attach to the status of a party, under the Statute and Rules of Court, or the general principles of procedural law".

23 CRAWFORD, Brownlie’s Principles of Public International Law, Oxford, 2012, p. 38.

24 Sobre a contribuição da ICJ no desenvolvimento do direito internacional dos direitos humanos ver AGNELLO, F. The dialogue between the International Court of Justice and specialized judicial and quasi-judicial bodies in matters relating to the protection of human rights in ARCARI, M.; BALMOND, L. LE DIALOGUE DES JURIDICTIONS DANS L'ORDRE JURIDIQUE INTERNATIONAL: ENTRE PLURALISME ET SECURITE JURIDIQUE. Napoli: Editoriale Scientifica, 2014.

25 Polish Postal Service in Danzig, PCIJ Ser. B - No. 11, 16 May 1925, p. 30: “[...] It is perfectly true that all the parts of a judgment concerning the points in dispute explain and complete each other and are to be taken into account in order to determine the precise meaning and scope of the operative portion. This is clearly stated in the award of the Permanent Court of Arbitration of October 14th, 1902, concerning the Pious Funds of the Californias, which has been repeatedly invoked by Danzig. The Court agrees with this statement. But it by no means follows that every reason given in a decision constitutes a decision; and it must be remembered that the Court of Arbitration applied the doctrine of res judicata because not only the Parties but also the matter in dispute was the same".

26 Nottebohm Case (Preliminary Objection), Judgment of 18 November 1953, in ICJ Report, 1953, p. 119.

27 Ver supra, nota 17.

28 Report of the Appellate Body, European Communities - Conditions for the Granting of Tariff Preferences to Developing Countries, WT/DS246/AB/R, 7 April 2004, para. 104.

29 Ibidem, para. 105.

30 Ibidem, para. 105, nota 220.

31 Report of the Appellate Body, United States - Anti-Dumping Act of 1916, WT/DS136/AB/R WT/DS162/AB/R, 28 August 2000, para. 54, nota 30.

32 Case IT-94-1-A-R77, 31 January 2000.

33 O Tribunal referiu-se ao caso Nuclear Tests (Australia v. France), Judgment of 20 December 1974, in ICJ Reports, 1974, pp. 259-260, para. 23; e ao caso Northern Cameroons, Judgment of 2 December 1963, in ICJ Reports, 1963, p. 29. See para. 13, nota 11.

34 Neste sentido, o Tribunal acrescentou que "As an international criminal court, the Tribunal must therefore possess the inherent power to deal with conduct which interferes with its administration of justice. The content of that inherent power may be discerned by reference to the usual sources of international law". Ver para. 13.

35 Fairén-Garbi and Solís-Corrales v. Honduras (merits), Judgment of 15 March 1989, para. 130. Ver também a sentença sobre questões preliminares emitida no mesmo caso em 26 de Junho de 1987, na qual a Corte, referindo-se aos casos Mavrommatis e Aegean Sea Continental Shelf, apontou em seu para. 38 que "[...] failure to observe certain formalities is not necessarily relevant when dealing on the international plane. What is essential is that the conditions necessary for the preservation of the procedural rights of the parties not be diminished or unbalanced and that the objectives of the different procedures be met".

36 Velásquez-Rodríguez v. Honduras (merits), Judgment of 29 July 1988, para. 163.

37 The "Grand Prince" Case (Belize v. France), Application for Prompt Release, Judgment of 20 April 2001, paras. 78-79. 
38 The M/V "Louisa" Case (Saint Vincent and the Grenadines v. Kingdom of Spain), Judgment of 28 May 2013.

39 Ibidem, para. 81, referindo-se ao caso Certain Norwegian Loans, Judgment of 6 July 1957, in ICJ Reports, 1957, p. 9, p. 23, e mencionando o caso Armed Activities on the Territory of the Congo (New Application: 2002) (Democratic Republic of the Congo v. Rwanda), Jurisdiction and Admissibility, Judgment of 3 February 2006, in ICJ Reports, 2006, p. 6, at p. 39, para. 88.

40 Ibidem, para. 95, referindo-se a Fisheries Jurisdiction (Spain v. Canada), Jurisdiction of the Court, Judgment of 4 December 1998, in ICJ Reports, 1998, p. 432, at p. 447, para. 29).

41 Ibidem, para. 99, referindo-se a Oil Platforms (Islamic Republic of Iran v. United States of America), Preliminary Objection, Judgment of 12 December 1996, in ICJ Reports, 1996, p. 803, p. 810, para. 16.

42 Ibidem, paras. 142-143, referindo-se a Prince von Pless Administration, Order of 4February 1933, P.C.I.J., Series A/B, No. 52, p. 11, at p. 14; Société commerciale de Belgique, Judgment, 1939, P.C.I.J., Series A/B, No. 78, p. 160, at p. 173; Certain Phosphate Lands in Nauru (Nauru v. Australia), Preliminary Objections, Judgment of 26 June 1992, in ICJ Reports, 1992, p. 265, para. 63 e para. 69 (' $[\ldots]$ the Tribunal concludes that these provisions are essential from the point of view of legal security and the good administration of justice'); Oil Platforms (Islamic Republic of Iran v. United States of America), Judgment of 6 November 2003, in ICJ Reports, 2003, p. 161, p. 213, para. 117.

43 ABI-SAAB, The International Court as a World Court, in LOWE, FITZMAURICE (eds.), Fifty Years of the International Court of Justice. Essays in Honour of Sir Robert Jennings, Cambridge, 1996, p. 3 ss.; Id., The Normalization of International Adjudication: Convergence and Divergences, in International Law and Politics, 2010, p. 9, de acordo com quem " [...] in laying down its own rules (or addressing their lacunae or ambiguities), an international judicial organ starts by looking at what the International Court does; unless there is a special reason to deviate from it that can be drawn from the Statute or the particular nature of the judicial organ". Ver, no mesmo sentido, CRAWFORD, Brownlie's Principles, cit., p. 41, que afirma que: “[...] [w] hilst it is correct to state that in international law 'every tribunal is a self-contained system (unless otherwise provided)', the Court's lengthy period of operation - throughout much of which it was the only international tribunal of any significance - has enabled it to lay down a body of procedural case-law which was and is a natural source of inspiration for later tribunal"; e GAFFNEY, Due Process, cit., pp. 1180-1181.

44 A prática de referir-se à jurisprudência da Corte Internacional de Justiça em questões processuais fundamentais parece corroborar a quarta suposição da assim chamada "teoria do precedente" desenvolvida por TERRIS, ROMANO AND SWIGART, The International Judge: An Introduction to the Men and the Women Who Decide the World's Cases, Oxford, 2007, p. 119 ss. Efetivamente, de acordo com este corolário "[...] specialized courts will consider, quote and defer to the ICJ on matters of general public international law". Portanto, pode ser afirmado que, na medida em que normas fundamentais do contencioso internacional são 'matter of general public international law", tal corolário é refletido na práxis relevante examinada anteriormente.

45 Ver GUILLAUME, The Use of Precedent, cit., p. 19, apontando que a CIJ "[...] has exclusively cited its own judgments and advisory opinions for a long time".

46 Ver supra, nota 2. Para uma análise desta opinião consultiva, ver DE BRABANDERE, Individuals in Advisory Proceedings before the International Court of Justice: Equality of the Parties and the Court's Discretionary Authority, in The Law and Practice of International Courts and Tribunals, 2012, p. 253 ss.; AMOROSO, The Judicial Activity of the International Court of Justice in 2012: A Year of Human Rights Cases, in Italian Yearbook of International Law, 2012, p. 223 ss.

47 Os outros quatro casos nos quais a Corte atuou como órgão de revisão neste campo são Judgments of the Administrative Tribunal of the I.L.O. upon complaints made against theU.N.E.S.C.O., Advisory Opinion of 23 October 1956, in ICJ Reports, 1956, p. 77 (doravante 1956 Advisory Opinion); Application for Review of Judgment No. 158 of the United Nations Administrative Tribunal, Advisory Opinion of 12 July 1973, in ICJ Reports, 1973, p. 166; Application for Review of Judgment No. 273 of 
the United Nations Administrative Tribunal, Advisory Opinion of 20 July 1982, in ICJ Reports, 1982, p. 325; Application for Review of Judgment No. 333 of the United Nations Administrative Tribunal, Advisory Opinion of 27 May 1987, in ICJ Reports, 1987, p. 18. Sobre este procedimento, ver, de maneira geral, KAIKOBAD, The International Court of Justice and Judicial Review. A Study of the Court Powers with Respect to Judgments of the ILO and UN Administrative Tribunals, The Hague, 2000; EISUKE, The Review and Repudiation of Judgments of International Administrative Tribunals, in Asian Yearbook of International Law, 1997, p. 175 ss.; GOMULA, The International Court of Justice and Administrative Tribunals of International Organizations, in Michigan Journal of International Law, 1991-1992, p. 83 fss.

$48 \mathrm{O}$ mecanismo de revisão era contemplado pelo Artigo 11 do Estatuto do TANU e foi definitivamente abolido pela Resolução da Assembléia Geral A/RES/50/54 de 11 de Dezembro de 1995. O inteiro sistema foi substituído em 2009 com o estabelecido de um mecanismo twotier de solução de disputa, consistindo no Tribunal de Controvérsias das Nações Unidas ( United Nations Dispute Tribunal) e o Tribunal de Apelação das Nações Unidas (United Nations Appeals Tribunal) (Resoluções da Assembléia Geral relativas: A/RES/61/261 of 4 April 2007, A/RES/62/228 of 22 December 2007 and A/RES/63/253 of 24 December 2008). Para uma visão geral, ver VARGIU, From Advisory Opinions to Binding Decisions: The New Appeal Mechanism of the UN System of Administration of Justice, in International Organization Law Review, 2010, p. 261 ss.

$49 \mathrm{O}$ artigo XII do Anexo ao Estatuto do TAOIT - o qual se aplica à organizações internacionais, como a IFAD, que tenham aceitado a jurisdição do TAOIT - descreve da seguinte forma: "1. In any case in which the Executive Board of an international organization which has made the declaration specified in article II. paragraph 5, of the Statute of the Tribunal challenges a decision of the Tribunal confirming its jurisdiction, or considers that a decision of the Tribunal is vitiated by a fundamental fault in the procedure followed, the question of the validity of the decision given by the Tribunal shall be submitted by the Governing Body, for an advisory opinion, to the International Court of Justice. 2. The opinion given by the Court shall be binding".

501956 Advisory Opinion, p. 86.

51 Como notou a Corte na Opinião Consultiva de 1956, p. 85, "[t] he special feature of this procedure is that advisory proceedings take the place of contentious proceedings which would not be possible under the Statute of the Court".

52 IFAD Advisory Opinion, para. 39 (referências internas omitidas).

531956 Advisory Opinion, p. 86.

54 IFAD Advisory Opinion, para. 44.

55 Ibidem.

56 Ibidem, para. 45.

57 Ibidem, para. 48.

58 Com relação ao papel e ao valor de comentários gerais adotados por órgãos de direitos humanos, ver BLAKE, Normative Instruments in International Human Rights Law: Locating the General Comment, Centre for Human Rights and Global Justice Working Paper, 2008. Válido mencionar aqui que, no caso Ahmadou Sadio Diallo (Guinea v. Congo), Judgment of 30 November 2010, in ICJ Reports, 2010, p. 639, para. 66, a ICJ, depois de referir-se General Comment No. 15: The position of Aliens under the Covenant of the Human Rights Committee, clarificou o seguinte: "[a] lthough the Court is in no way obliged, in the exercise of its judicial functions, to model its own interpretation of the Covenant on that of the Committee, it believes that it should ascribe great weight to the interpretation adopted by this independent body that was established specifically to supervise the application of that treaty. The point here is to achieve the necessary clarity and the essential consistency of international law, as well as legal security, to which both the individuals with guaranteed rights and the States obliged to comply with treaty obligations are entitled". 
59 Com efeito a CIJ diversas vezes abordou questões relativas a direitos humanos e contribuiu ao desenvolvimento deste campo. Para uma visão geral, ver BEDI, The Development of Human Rights Law by the Judges of the International Court of Justice, Oxford, 2007.

60 Esta expressão é de DUPUY, VIÑUALES, The Challenge of "Proliferation": AnAnatomy of the Debate, in ROMANO, ALTER, SHANY (eds.), The Oxford Handbook of International Adjudication, Oxford, 2013, p. 150.

621er DUPUY, VIÑUALES, The Challenge, cit. Em relação ao fenômeno da integração de valores emergentes do direito internacional dos direitos humanos e sobre direito dos investimentos, ver PUMA, Human rights and investment law: Attempts at harmonization through a difficult dialogue between arbitrators and human rights Tribunals, in ARCARI, BALMOND, cit.

62 Sobre o argumento de amicus curiae no contencioso internacional, ver BARTHOLOMEUSZ, The amicus curiae before International Courts and Tribunals, in BIANCHI (ed.), Non-State Actors and International Law, Dartmouth, 2009, p. 253 ss.; ASCENSIO, Lamicus curiae devant les juridictions internationales, in Revue Générale de Droit International Public, 2001, p. 897 ss.

$63 \mathrm{O}$ Artigo 34, para. 2 do Estatuto prevê que "[t]he Court, subject to and in conformity with its Rules, may request of public international organizations information relevant to cases before it, and shall receive such information presented by such organizations on their own initiative". No que se refere aos procedimentos Consultivos, também Estados podem oferecer informação sobre a questão. Neste sentido, ver o Artigo 66, para. 2 do Estatuto, de acordo com o qual "[t]he Registrar shall also, by means of a special and direct communication, notify any state entitled to appear before the Court or international organization considered by the Court, or, should it not be sitting, by the President, as likely to be able to furnish information on the question, that the Court will be prepared to receive, within a time-limit to be fixed by the President, written statements, or to hear, at a public sitting to be held for the purpose, oral statements relating to the question".

64 Ver o Artigo 50 do Estatuto, e os Artigos 62, 63 e 67 do Regulamento da CIJ.

65 A mesma consideração é aplicável no que se refere à posição de Estados que, em procedimentos contenciosos, não estão formalmente permitidos de participar como amici curiae. Sobre este ponto, ver PALCHETTI, Opening the International Court of Justice to Third States: Intervention and Beyond, in Max Planck Yearbook on United Nations Law, 2002, pp. 167-168.

66 Ibidem, pp. 168-170, "[t]he Court may avail itself of the amicus curiae procedure as an additional means for collecting evidence. In this sense, it seems tenable that the power to acquire evidence proprio motu includes also the possibility of accepting and evaluating views submitted by third states as amici curiae".

67 No que se refere a CEDH, ver o Artigo 36, para. 2 da Convenção e a Regra 44 do Regulamento da Corte. Para a CIDH, ver o Artigo 62, para. 3 das Regras de Procedimento.

\section{Referencias Bibliográficas}

AGNELLO, F. The dialogue between the International Court of Justice and specialized judicial and quasi-judicial bodies in matters relating to the protection of human rights in ARCARI, M.; BALMOND, L. LE DIALOGUE DES JURIDICTIONS DANS L'ORDRE JURIDIQUE INTERNATIONAL: ENTRE PLURALISME ET SECURITE JURIDIQUE. Napoli: Editoriale Scientifica, 2014.

AMOROSO, The Judicial Activity of the International Court of Justice in 2012: A Year of Human Rights Cases, in Italian Yearbook of International Law, 2012, p. 223 ss. 
Application for Review of Judgment No. 158 of the United Nations Administrative Tribunal, Advisory Opinion of 12 July 1973, in ICJ Reports, 1973.

Application for Review of Judgment No. 273 of the United Nations Administrative Tribunal, Advisory Opinion of 20 July 1982, in ICJ Reports, 1982.

Application for Review of Judgment No. 333 of the United Nations Administrative Tribunal, Advisory Opinion of 27 May 1987, in ICJ Reports, 1987.

Armed Activities on the Territory of the Congo (New Application: 2002) (Democratic Republic of the Congo v. Rwanda), Jurisdiction and Admissibility, Judgment of 3 February 2006, in ICJ Reports, 2006.

BARTHOLOMEUSZ, The amicus curiae before International Courts and Tribunals, in BIANCHI (ed.), Non-State Actors and International Law, Dartmouth, 2009.

ASCENSIO, Lamicus curiae devant les juridictions internationales, in Revue Générale de Droit International Public, 2001.

BEDI, The Development of Human Rights Law by the Judges of the International Court of Justice, Oxford, 2007.

BLAKE, Normative Instruments in International Human Rights Law: Locating the General Comment, Centre for Human Rights and Global Justice Working Paper, 2008.

BROWN, The Cross-Fertilization of Principles Relating to Procedure and Remedies in the Jurisprudence of International Courts and Tribunals, in Loyola of Los Angeles International and Comparative Law Review, 2008.

CANNIZZARO, Il rilievo degli accordi esterni nell'interpretazione degliaccordi OMC, in LIGUSTRO, SACERDOTI (eds.), Problemi e tendenze del diritto internazionale dell'economia. Liber amicorum in onore di Paolo Picone, Napoli, 2011, p. 513 ss.

Case IT-94-1-A-R77, 31 January 2000.

Certain Norwegian Loans, Judgment of 6 July 1957, in ICJ Reports, 1957.

Certain Phosphate Lands in Nauru (Nauru v. Australia), Preliminary Objections, Judgment of 26 June 1992, in ICJ Reports, 1992.

CHENG, General Principles of Law as Applied by International Courts and Tribunals, London, 1953, p. 257 ss.;

Corte Internacional de Justiça, Judgment No. 2867 of the Administrative Tribunal of the International Labour Organization upon a Complaint Filed against the International Fund for Agricultural Development, Advisory Opinion of 1 February 2012, in ICJ Reports, 2012. 
CRAWFORD, Brownlie’s Principles of Public International Law, Oxford, 2012, p. 38.

(Resoluções da Assembléia Geral relativas: A/RES/61/261 of 4 April 2007, A/RES/62/228 of 22 December 2007 and A/RES/63/253 of 24 December 2008).

DE BRABANDERE, Individuals in Advisory Proceedings before the International Court of Justice: Equality of the Parties and the Court's Discretionary Authority, in The Law and Practice of International Courts and Tribunals, 2012.

DUPUY, VIÑUALES, The Challenge of "Proliferation": AnAnatomy of the Debate, in ROMANO, ALTER, SHANY (eds.), The Oxford Handbook of International Adjudication, Oxford, 2013.

PUMA, Human rights and investment law: Attempts at harmonization through a difficult dialogue between arbitrators and human rights Tribunals, in ARCARI, BALMOND.

ECtHR, Mamatkulov and Abdurasulovic v. Turkey, Applications Nos. 46827/99 and 46951/99, Judgment of 6 February 2003.

ECtHR, paras. 102-105, citando LaGrand (Germany v. United States), Judgment of 27 June 2001, in ICJ Reports, 2001.

EISUKE, The Review and Repudiation of Judgments of International Administrative Tribunals, in Asian Yearbook of International Law, 1997.

Fisheries Jurisdiction (Spain v. Canada), Jurisdiction of the Court, Judgment of 4 December 1998, in ICJ Reports, 1998, p. 432, at p. 447.

FITZMAURICE (eds.), Fifty Years of the International Court of Justice. Essays in Honour of Sir Robert Jennings, Cambridge, 1996, p. 389;

GAFFNEY, Due Process in the World Trade Organization: The Need for Procedural Justice in the Dispute Settlement System, in American University International Law Review, 1999, p. 1179-1180;

GOMULA, The International Court of Justice and Administrative Tribunals of International Organizations, in Michigan Journal of International Law, 1991-1992.

GUILLAUME, The Use of Precedent by International Judges and Arbitrators, in Journal of International Dispute Settlement, 2011, p. 9 ss.; Shaw, International Law, Cambridge, 2008 .

Judgments of the Administrative Tribunal of the I.L.O. upon complaints made against theU.N.E.S.C.O., Advisory Opinion of 23 October 1956, in ICJ Reports, 1956.

KAIKOBAD, The International Court of Justice and Judicial Review. A Study of the Court Powers with Respect to Judgments of the ILO and UN Administrative Tribunals, The Hague, 2000; 
KOLB, General Principles of Procedural Law in ZIMMERMANN, TOMUSCHAT, and OELLERS-FRAHM (eds), The Statute of the International Court of Justice: A Commentary, Oxford, 2012, p. 871 ss.

KOLB, La maxime de la "bonne administration de la justice" dans la jurisprudence internationale, in L'observateur des Nations Unies, 2009, pp. 7-8.

LOWE, FITZMAURICE (eds.), Fifty Years of the International Court of Justice. Essays in Honour of Sir Robert Jennings, Cambridge, 1996.

LOWE, FITZMAURICE., The Normalization of International Adjudication:

Convergence and Divergences, in International Law and Politics, 2010.

MANI, International Adjudication: Procedural Aspects, The Hague, 1980, p. 12;

MATSCHER, Standing Before International Courts and Tribunals, in EPIL, 1981 p.191;

MILLER, An International Jurisprudence? The Operation of "Precedent" Across

International Tribunals, in Leiden Journal of International Law, 2002, p. 488

NEGRI, I principi generali del processo internazionale nella giurisprudenza della Corte internazionale di giustizia, Napoli, 2002, pp. 32-33;

Nottebohm Case (Preliminary Objection), Judgment of 18 November 1953, in ICJ Report, 1953.

Oil Platforms (Islamic Republic of Iran v. United States of America), Preliminary Objection, Judgment of 12 December 1996, in ICJ Reports, 1996, p. 803, p. 810.

PALCHETTI, Opening the International Court of Justice to Third States: Intervention and Beyond, in Max Planck Yearbook on United Nations Law, 2002, pp. 167-168.

PALOMBINO, Judicial Economy and the Limitation of the Scope of the Decision in International Adjudication, in Leiden Journal of International Law, 2010, p. 910 ss.

Polish Postal Service in Danzig, PCIJ Ser. B - No. 11, 16 May 1925.

Prince von Pless Administration, Order of 4February 1933, P.C.I.J., Series A/B, No. 52.

REINISCH, The Proliferation of International Dispute Settlement Mechanism: The Threat of Fragmentation vs. the Promise of a More Effective System? Some Reflections From the Perspective of Investment Arbitration, in BUFFARD, CRAWFORD, PELLET, WITTICH (eds.), International Law Between Universalism and Fragmentation.

Festschrift in Honour of Gerhard Hafner, Leiden/Boston, 2008, p. 123. Ver igualmente ROSENNE, The Perplexities of Modern International Law, Leiden, 2004.

Report of the Appellate Body, European Communities - Conditions for the Granting of Tariff 
Preferences to Developing Countries, WT/DS246/AB/R, 7 April 2004.

Report of the Appellate Body, United States - Anti-Dumping Act of 1916, WT/DS136/ AB/R - WT/DS162/AB/R, 28 August 2000, para. 54, nota 30.

ROMANO, Deciphering the Grammar of the International Jurisprudential Dialogue, in International Law and Politics, 2009, pp. 759-760

SCHLESINGER, Research on the General Principles of Law Recognized by Civilized Nations, in American Journal of International Law, 1957, p. 736;

SERENI, Principi generali di diritto e processo internazionale, Milano, 1955, passim;

SHAHABUDDEN, Precedent in the World Court, Cambridge, 1996.

SHAHABUDDEN, Precedent in the World Court, cit., p. 2, grifo adicionado.

Société commerciale de Belgique, Judgment, 1939, P.C.I.J., Series A/B, No. 78.

TARUFFO, Precedente e giurisprudenza, Napoli, 2007, p. 32 (Tradução em inglês realizada pelo autor).

TERRIS, ROMANO AND SWIGART, The International Judge: An Introduction to the Men and the Women Who Decide the World's Cases, Oxford, 2007.

The "Grand Prince" Case (Belize v. France), Application for Prompt Release, Judgment of 20 April 2001, paras. 78-79.

The M/V "Louisa” Case (Saint Vincent and the Grenadines v. Kingdom of Spain), Judgment of 28 May 2013.

THIRLWAY, Procedural Law and the International Court of Justice, in LOWE.

VARGIU, From Advisory Opinions to Binding Decisions: The New Appeal Mechanism of the UN System of Administration of Justice, in International Organization Law Review, 2010, p. 261 ss.

Velásquez-Rodríguez v. Honduras (merits), Judgment of 29 July 1988.

WEINIGER and SCHREUER, Conversations across Cases: Is there a Doctrine of Precedent in Investment Arbitration?, in Transnational Dispute Management, 2008;

ZAMMIT BORDA, Precedent in International Criminal Courts and Tribunals, in Cambridge Journal of International and Comparative Law, 2013, p. 288. 


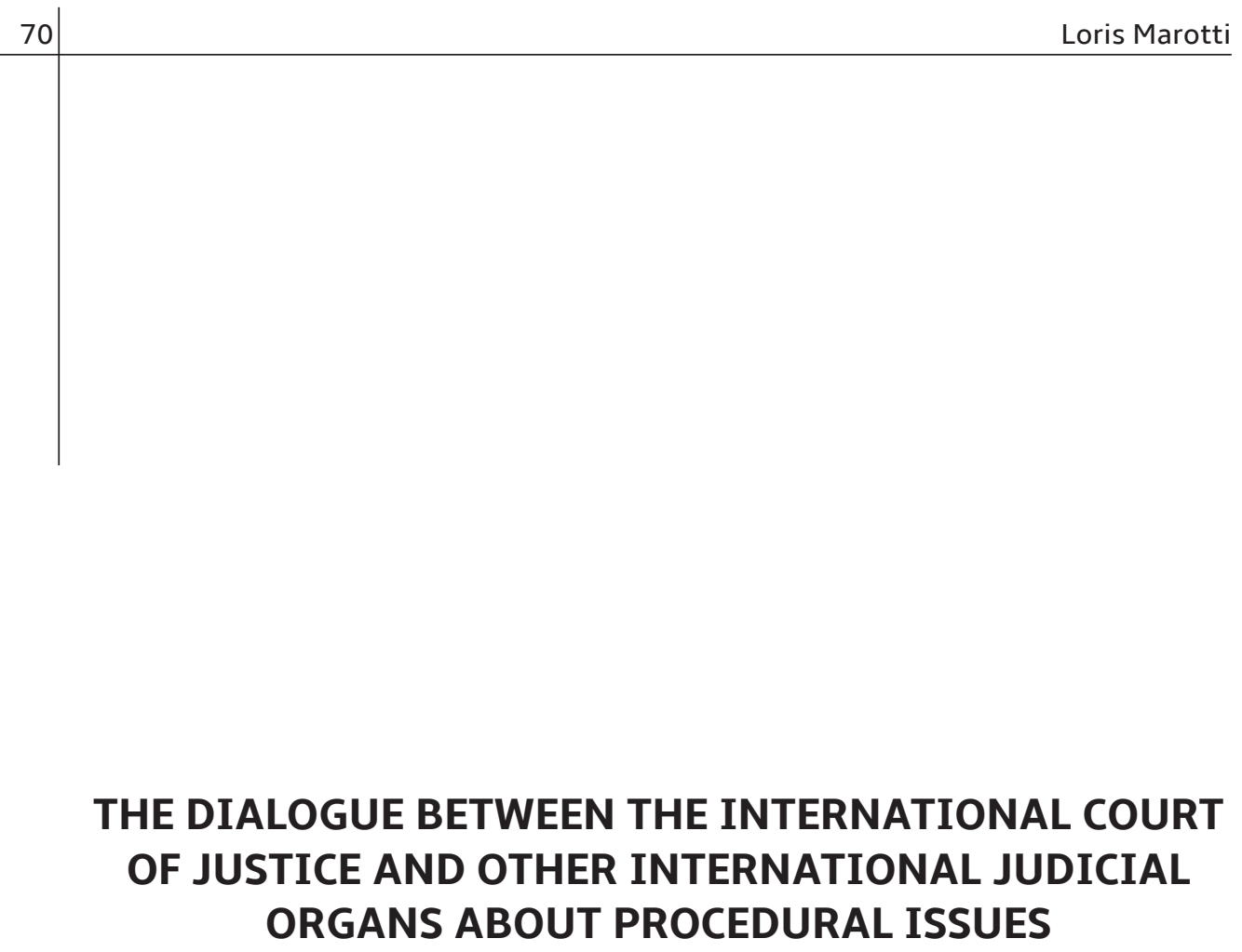

\section{Resumo}

Este trabalho analisa e discute o diálogo judicial relacionado a questões processuais e, em particular, evidencia o essencial papel desempenhado pela Corte Internacional de Justiça (CIJ) na determinação e no desenvolvimento de princípios e regras de processo judicial internacional. A primeira parte deste trabalho irá afrontar alguns aspectos gerais relativos à prática de "diálogo judicial" no contencioso internacional e as fontes do direito processual internacional. A segunda parte é uma análise da práxis de diversos órgãos judiciais internacionais que "dialogaram" com a CIJ em questões processuais. A terceira e última parte focará no posicionamento tomado pela CIJ em relação à determinação e interpretação de princípios processuais e, à luz da abordagem adotada pela Corte na Opinião Consultiva IFAD, traçar os possíveis desenvolvimentos emergentes deste tipo de abordagem.

Palavras-chave: Corte Internacional de Justiça. Contencioso Internacional. Opinião Consultiva IFAD. 


\begin{abstract}
This paper analyses and discusses the judicial dialogue relating to procedural matters and, in particular, highlights the pivotal role played by the International Court of Justice (ICJ) in determining and developing principles and rules of judicial procedure. The first part of this paper will address some general aspects regarding the practice of "judicial dialogue" in international adjudication and the sources of international procedural law. The second part is an analysis of the practice of several international judicial bodies which have "dialogued" with the ICJ in addressing procedural issues. The third and last part will focus on the attitude taken by the ICJ in relation to the determination and interpretation of procedural principles and, in the light of the approach adopted by the Court in the IFAD Advisory Opinion, will trace possible developments arising from that kind of approach.
\end{abstract}

Keywords: International Court of Justice. International Adjudication. IFAD Advisory Opinion.

Recebido em agosto de 2018. Aprovado em novembro de 2018. 